\title{
BMJ Open Identifying values and preferences around the choice of analgesia for patients with acute trauma pain in emergency and prehospital settings: using group concept mapping methodology
}

\author{
Kristina Tomra Nielsen (1) ,,2 Marianne Uggen Rasmussen (1) , \\ Anders Foehrby Overgaard, ${ }^{2}$ Louise Klokker, ${ }^{2}$ Robin Christensen, ${ }^{2,3}$ \\ Eva Ejlersen Wæhrens ${ }^{2,4}$
}

To cite: Nielsen KT, Rasmussen MU, Overgaard AF, et al. Identifying values and preferences around the choice of analgesia for patients with acute trauma pain in emergency and prehospital settings: using group concept mapping methodology. BMJ Open 2020;10:e031863. doi:10.1136/ bmjopen-2019-031863

- Prepublication history and additional material for this paper are available online. To view these files, please visit the journal online (http://dx.doi. org/10.1136/bmjopen-2019031863).

KTN and MUR are co-first authors.

Received 23 May 2019 Revised 15 January 2020 Accepted 10 February 2020

D) Check for updates

(C) Author(s) (or their employer(s)) 2020. Re-use permitted under CC BY-NC. No commercial re-use. See rights and permissions. Published by BMJ.

For numbered affiliations see end of article.

\section{Correspondence to} Dr Marianne Uggen Rasmussen; marianne.uggen.rasmussen@ regionh.dk

\section{ABSTRACT}

Objectives The main study aim was to examine the applicability of a novel method to assess the criterion of values and preferences within the Grading of Recommendation, Assessment, Development and Evaluation evidence to decision framework. The group concept mapping (GCM) approach was applied to identify, organise and prioritise values and preferences in the example of health professionals' choice of analgesia for patients with acute trauma pain.

Setting Prehospital and emergency care centres in the Nordic countries of Denmark, Norway, Sweden, Finland and Iceland.

Participants Acute care health professionals with qualifications to administer analgesic agents to patients in emergency and prehospital settings, including advanced ambulance assistants, rescue officers, paramedics, emergency physicians and emergency nurses, participated in an online survey in which statements were generated $(n=40)$ and structured $(n=11)$ and finally analysed and interpreted in a validation meeting $(\mathrm{n}=4)$.

Results Using GCM, ideas were generated and structured through online participation. Results were interpreted at a validation meeting. In total, 111 unique ideas were identified and organised into seven clusters: drug profile, administration, context, health professionals' preferences and logistics, safety profile, patient's medical history and acute clinical situation.

Conclusions Based on GCM, a conceptual model was developed, and values and preferences around choice of analgesia in emergency care were revealed. Health professionals within acute care can apply the conceptual model to support their decision-making when choosing the best available treatment for pain for their patients in emergency care.

\section{INTRODUCTION}

Health professionals in emergency care are constantly making informed decisions when
Strengths and limitations of this study

- Group concept mapping represents a mixed methods approach with true integration of qualitative and quantitative data

- The process values the voice and involvement of the participants, who are involved in generating data, and in data analyses and validation of the results.

- All five Nordic countries with participants from different disciplines were represented in the brainstorming, sorting and rating phases.

- The online participation yielded a large number of statements out of which several were redundant, indicating data saturation.

- This study was possibly limited by a smaller sample size than anticipated.

choosing among the different available treatment options for their patients. In emergency situations, the treatment of acute trauma pain is of primary concern. Health professionals dealing with acute patients are typically ambulance crew, care assistants, paramedics, emergency nurses and medical doctors. Treatment of acute pain entails specific requirements to the applied analgesic agent, including easy administration, minimal equipment and fast onset of pain relief. ${ }^{1}$ This adds to the generic demands for healthcare treatments, which are: adequate evidence of effect, an acceptable balance between benefits and harms, accordance with the users' (patients and health professionals) values and preferences, and favourable cost-effectiveness. ${ }^{2}$ Thus, healthcare decision-making is complex with many factors to consider. 
Explicit and transparent systems for decision-making can ensure that all important criteria are considered and that the clinicians' decisions are informed by the best available research evidence. Health professionals can rely on recommendations from clinical practice guidelines in their decision-making. Guidelines are based on expert groups' consideration of the evidence within a field, and recommendations are made based on trade-offs between desirable and undesirable consequences of alternative management strategies. The Grading of Recommendation, Assessment, Development and Evaluation (GRADE) evidence to decision framework represents a systematic and transparent system that health professionals can use to make well-informed healthcare choices. ${ }^{3}$ Within the GRADE framework, besides the balance between benefits and harms, criteria with regard to the quality of the evidence, the cost/burden to society and the values and preferences of the stakeholders such as patients and health professionals should be considered in the recommendations. The values and preferences criterion within GRADE is defined as the relative importance the population of interest, such as patients or stakeholders, places on the main outcome in question. ${ }^{4}$

Although these criteria are of equal importance in the decision-making process when substantiating clinical recommendations and guidelines, values and preferences may have been overlooked in the process, possibly due to a lack of consensus on how to assess this criterion. Research evidence per se cannot tell us which treatment is best for patients. Incorporating patients and professionals' preferences into treatment recommendations has been viewed as the next step in guideline development, but so far this rarely occurs in practice, although studies have been conducted on how to investigate patients' values and preferences using various qualitative methods. ${ }^{5-8}$

Systematic reviews have been conducted to investigate patients ${ }^{9}$ and other stakeholders ${ }^{10}$ values and preferences with regard to treatment options in different settings. These reviews are based on studies of different designs including cross-sectional interviews, surveys and randomised controlled trials. Qualitative open-ended interviews, on the other hand, have also been conducted to investigate different stakeholders' values and preferences, ${ }^{11}$ and another systematic review has included only qualitative studies to investigate patients' values and preferences. ${ }^{12}$ Still, the area of values and preferences is underexplored, and the methodology on how to assess this criterion to substantiate recommendations within GRADE is not yet fully developed. ${ }^{13}$

In this study, we apply group concept mapping $(\mathrm{GCM})^{14-16}$ as a novel approach to identifying, organising and prioritising values and preferences among stakeholders, using the example of health professionals' choosing of analgesia for patients with acute trauma pain in emergency and prehospital settings. The GCM method was developed in the early 1980s to have various stakeholders generate and structure ideas on a specific topic. Through the GCM process, concepts are visualised through the creation of maps, where ideas on a specific topic are depicted according to their internal relations. GCM is based on a mixed methods approach by using qualitative generation of data (ie, ideas on a specific topic) combined with statistical analyses to support the structuring of data. ${ }^{14}$ The GCM method has previously been applied to gain various perspectives related to planning and evaluation within the healthcare system, ${ }^{17-21}$ including gaining perspectives of various healthcare professionals. ${ }^{1722} 23$

\section{MATERIALS AND METHODS Procedure}

To gain broad perspectives on values and preferences associated with health professionals' choice of analgesia in emergency and prehospital settings, GCM was applied. ${ }^{1416}$ The GCM approach involves key stakeholders (eg, patients and/or professionals) in several steps of the process, including generation of data, analyses and interpretation of the results. The following phases are included in the GCM process: (1) preparing for GCM, (2) generating the ideas (brainstorming), (3) structuring the statements (sorting and rating), (4) GCM analysis (data analysis), (5) interpreting the map, and (6) utilisation. ${ }^{14}$ Phases 2 and 3 can be conducted in face-to-face group sessions and/or by individual online participation. ${ }^{14}$ Online participation was applied in the present study.

A pooled study analysis based on GCM studies ${ }^{24}$ has indicated high reliability (eg, based on sorting and rating reliability estimates), high external representational validity (ie, to which extent the conceptualised model reflects what it is intended to reflect) and high internal representational validity (ie, to which extent the conceptualised model represents the judgements of the participants during the process of organising the data). Further, the sorting and rating completion percentages generally were high. ${ }^{24}$

\section{Participants}

Health professionals from the Nordic countries (Denmark, Norway, Sweden, Finland and Iceland), who treat patients with acute pain in emergency and prehospital settings, were recruited from November 2017 to January 2018. Initially, potential key participants were identified through networks of emergency care. A total of 63 health professionals representing different disciplines across the five Nordic countries were invited to participate in the study by email. This purposeful chain sampling strategy was applied to ensure a diverse sample of professions across countries as a means to substantiate data saturation. Subsequently, snowball sampling was applied, as invited participants were asked to forward the invitation to other health professionals in their multidisciplinary acute care network, who also were asked to forward the invitation in their professional network. ${ }^{25}$

Included were health professionals with qualifications to administer analgesic agents in emergency and prehospital settings such as advanced ambulance 
assistants, rescue officers, paramedics, emergency physicians, emergency nurses, working in the Nordic countries and able to read and write in English. To ensure a representative sample, we sought to include as many participants as possible through the snowball sampling strategy, and to make sure that participants from different disciplines within acute care were represented and took part in throughout all phases in the GCM process.

\section{Patient and public involvement}

There were no patients involved in this study.

\section{GCM: data generation}

The previously described phases in the process of GCM will serve as a structure describing the procedures in the study.

\section{Preparing for GCM}

Before initiating the data collection, a seeding question was formulated:

When making your choice of analgesia in emergency and pre-hospital settings in patients with acute trauma pain, what do you value?

\section{Generating the ideas (brainstorming)}

Initially, invited participants received an email with information about the study and a link to SurveyXact online survey system. ${ }^{26}$ Participants were then asked to answer demographic questions (age, gender, country of residence, profession, number of years in the field of emergency and current position). Next, the participants were asked to think as broadly as possible to generate ideas in response to the seeding question.

Each participant typed his/her responses in the form of statements (sentences or single words). There were no restrictions on the number of statements, but there should be separate statements for each issue. Statements were to be given in English, but native languages were also permitted to avoid language limitations. The participants were not able to see the statements typed in by others.

\section{Structuring the statements (sorting and rating)}

Participants were invited to the next phase by email containing a link to an online system developed for the purpose, CS Global Max. ${ }^{27}$ In CS Global Max, the participants were again asked to answer questions on demographics. Further, the participants were presented to the total number of statements and asked to organise all statements into piles, in any way that made sense to him/her. The only two rules were: (A) there must be more than one pile and (B) there must be fewer piles than the number of statements. Each participant was also asked to label each pile of statements and based on the seeding question the participants were asked to rate the importance of each statement using a 4-point ordinal scale: (1) 'Not at all important', (2) 'Somewhat unimportant', (3) 'Somewhat important', and (4) 'Very important'.

\section{Data analysis}

Demographic data

Descriptive statistics were performed using Microsoft Excel software. ${ }^{28}$ Demographic data on age and number of years in the field of emergency were presented based on median and range due to lack of normal distribution of the data. Data on gender, country of residence, profession and worker role were presented in percentages.

\section{GCM: data analysis}

Data were analysed at several steps during the process. First, the ideas generated by online participants were consolidated; two members of the research group identified redundant ideas (ie, ideas with exactly the same wording) individually. Next, the researchers met and discussed their findings. Based on consensus, redundant ideas were removed, minor linguistic revisions were made to clarify the meaning. The remaining ideas were then imported into CS Global Max. During the next phase, statements were sorted and rated by the participants. Based on the sorting and ratings, a multidimensional scaling analysis and cluster analyses were performed. ${ }^{14}$ Within the multidimensional scaling analysis, the statistic used to indicate 'goodness of fit' is called the stress value. A low stress value $<0.39$ indicates congruence between the raw data and processed data. ${ }^{24}$

Based on cluster analyses, a cluster rating map was generated. Several cluster solutions were applied and the one that best matched the data (ie, the cluster solution representing sufficient details on the topic) was selected. The clusters were labelled based on the label names provided by the participants during phase 2 of the GCM process. The importance of the ideas in each cluster was depicted by the height of the clusters, due to how many layers the cluster consisted of. For data to be included, the participant had to have sorted more than $75 \%$ of the statements and had to have left less than five statements unrated. The ratings of importance for each idea were based on a 1-4 ordinal scale and the ratings were, therefore, presented as medians. The number of ideas rated to be 'Very important' (median equal to 4) and 'Somewhat important' (median equal to 3 ) was identified.

\section{Interpreting the map (validation meeting and postworkshop analysis)}

A representative subset of participants was invited to a workshop to interpret and validate the results. In the workshop, participants were introduced to the cluster rating map, provided with an overview of clusters and statements, and asked to individually (A) determine if each statement was placed in the right cluster, (B) consider the number of clusters, and $(\mathrm{C})$ consider if the cluster labels illustrated the theme of the cluster. Next, the participants engaged in small group discussions around their reflections. Last, all suggestions were discussed in 
the large group and consensus was sought regarding cluster names, content and numbers of clusters. Thus, consensus was used as criteria for making changes. Based on the cluster rating map and the input from the validation meeting, the research team developed a conceptual model that illustrated the themes identified in relation to values and preferences around choice of analgesia in emergency and prehospital settings in patients with acute trauma pain values and preferences.

\section{Utilisation}

The conceptual model provided information on the themes related to values and preferences around choice of analgesia in emergency and prehospital settings in patients with acute trauma pain values and preferences. Further, the model provided information on the importance of the themes and how the themes were inter-related.

\section{Ethics}

All participants received written information before they agreed to participate and provided informed consent. All participants were informed about their rights and that participation was voluntarily, and withdrawal of their consent could take place at any time without consequences. According to Danish legislation, projects with survey data collection do not require ethical approval. ${ }^{29}$ Authentication that no ethical approval was required was obtained from the independent local ethics committee (Journal No H-18057398). Approval from the Danish Data Protection Agency was obtained (Journal No 2012-58-0004).

\section{RESULTS}

Using GCM, participants representing variation across professions and nationalities were recruited to identify, organise and prioritise values and preferences in the example of health professionals' choice of analgesia for patients with acute trauma pain. Ideas were generated representing a broad perspective on the topic. Sortings and ratings resulted in a cluster rating map which became the foundation for developing a conceptual model. In the following sections, the demographic data of the participants, the GCM data and the conceptual model are presented.

\section{Participants: demographic data}

Figure 1 flow chart presents the study flow and illustrates the number of participants, ideas and clusters in the different phases. During the phases of generating ideas and structuring statements, health professionals from all five Nordic countries participated. Table 1 presents the demographic data of the participants in the different study phases. A total of 40 health professionals participated in generating ideas and 13 participated in structuring the statements, out of whom 11 participants sorted and labelled the statements and all 13 rated the importance. All 11 participants sorted $100 \%$ of the statements. Nine of the 13 , who rated the importance, left between 1 and 13 statements unrated $(n=37)$. As the proportion of unrated statements for each participant was below 25\%, no data were excluded. Finally, five health professionals from Denmark, Norway and Finland participated in the validation meeting.

\section{GCM data}

A total of 151 statements were generated. After removing redundant ideas, 111 unique statements remained and were included in the initial cluster rating map (figure 1).

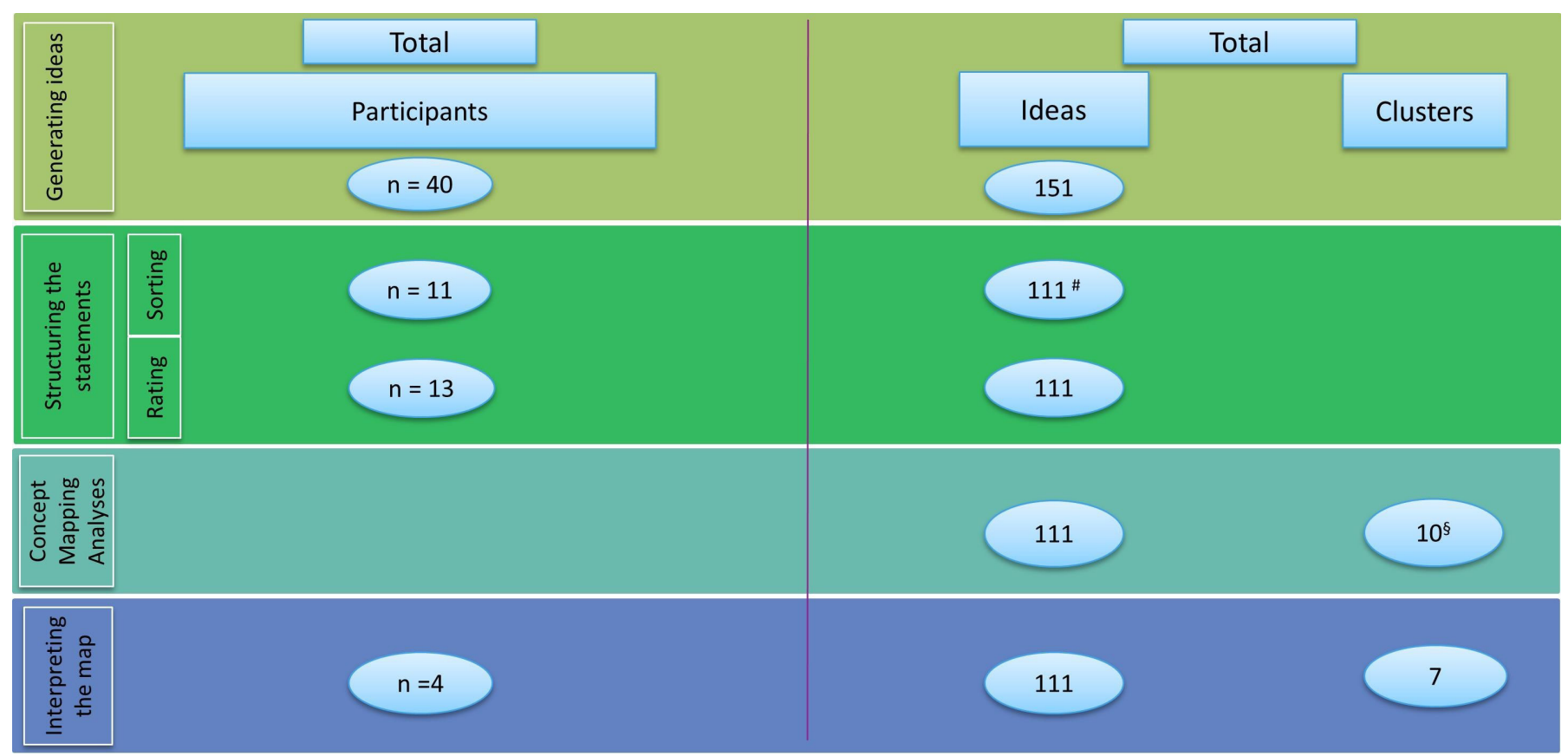

Figure 1 Flow chart illustrating the number of participants, ideas and clusters in the different phases. \#Unique ideas remaining after removal of redundancies. §A solution with 10 clusters was chosen out of cluster solutions with 8, 9, 10, 11 and 12 clusters. 
Table 1 Demographic data of the participants

\begin{tabular}{|c|c|c|c|}
\hline & $\begin{array}{l}\text { Generating the ideas } \\
(n=40)\end{array}$ & $\begin{array}{l}\text { Structuring the statements } \\
(n=13)\end{array}$ & $\begin{array}{l}\text { Interpreting the map } \\
(n=4)\end{array}$ \\
\hline Gender female, $\mathrm{n}(\%)$ & $7(18)$ & $3(23)$ & $1(25)$ \\
\hline \multicolumn{4}{|l|}{ Country of workplace, $\mathrm{n}(\%)$} \\
\hline Denmark & $11(28)$ & $3(23)$ & $1(25)$ \\
\hline Norway & $9(23)$ & $2(15)$ & $2(50)$ \\
\hline Sweden & $1(3)$ & $1(8)$ & \\
\hline \multicolumn{4}{|l|}{ Position, n (\%) } \\
\hline Advanced ambulance assistant & $2(5)$ & & \\
\hline Emergency nurse & $5(13)$ & & \\
\hline Other & $4(10)$ & & $1(25)$ \\
\hline Years of experience, median (range) & $13(2-37)$ & $20(6-25)$ & $20.5(11-25)$ \\
\hline
\end{tabular}

Of the 111 statements, $13(12 \%)$ were rated to be 'Very important' (median=4) and 76 statements $(68 \%)$ were rated to be 'Somewhat important' (median=3). Cluster solutions with 8-12 clusters were applied. The cluster solution with 10 clusters was chosen as this solution matched the data best by initially providing sufficient details on the topic. The 10 clusters are presented in a cluster rating map (figure 2). Each cluster contained between 4 and 28 ideas with varying importance, which is depicted by the height of clusters. The most important ideas were placed in the highest clusters. The multidimensional scaling analysis revealed a stress value of 0.22 .

Discussions at the face-to-face validation meeting lead to some clusters being collapsed. More specifically, clusters 8 and 9 were collapsed and clusters 5 and 6 were collapsed. All the statements in cluster 7 were moved into other clusters, hence, reducing the total number of clusters from 10 to 7 . The participants agreed on the location of the majority $(n=92,82.8 \%)$ of the statements, but $19(17.2 \%)$ statements were moved from one cluster to another. Further, the participants at the validation meeting suggested minor changes to all but one of the labels. This resulted in the following seven clusters: $d r u g$ profile, administration, context, health professionals' preferences and logistics, safety profile, patient's medical history and acute clinical situation containing between 7 and 37 statements.

The final clusters and all the included statements are presented in online supplementary table 1. Based on

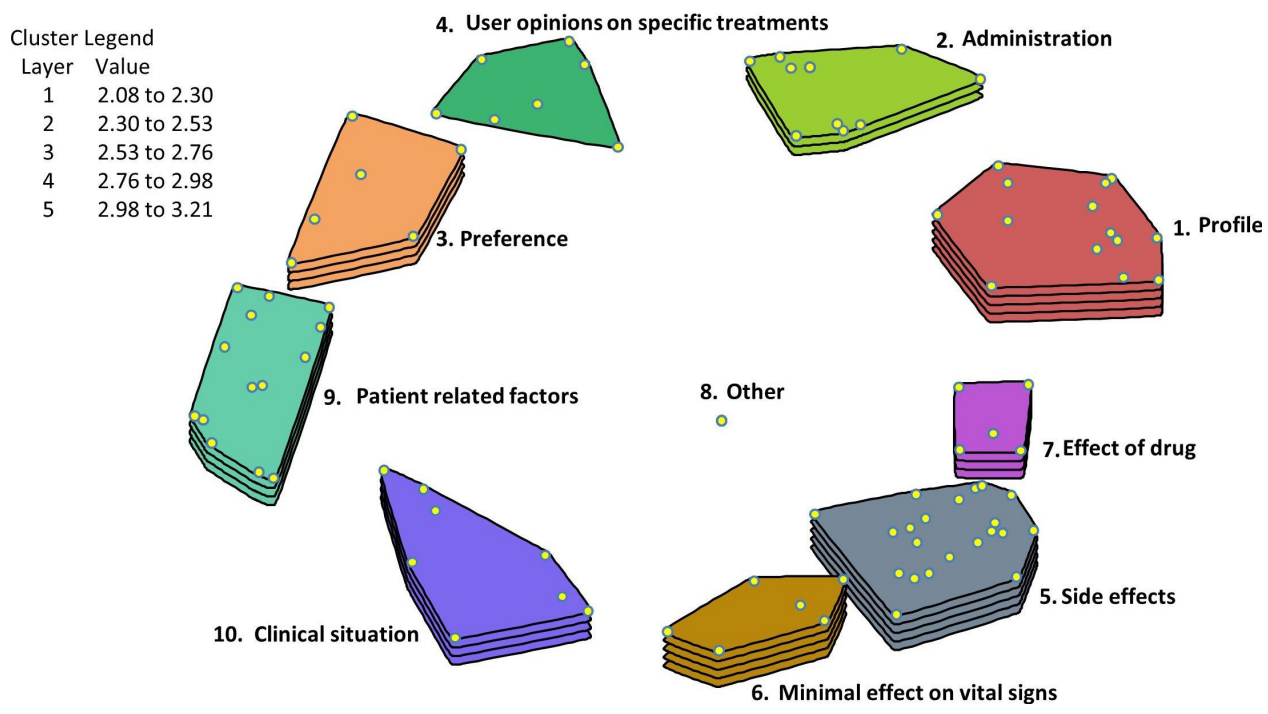

Figure 2 Cluster rating map with 10 clusters. 


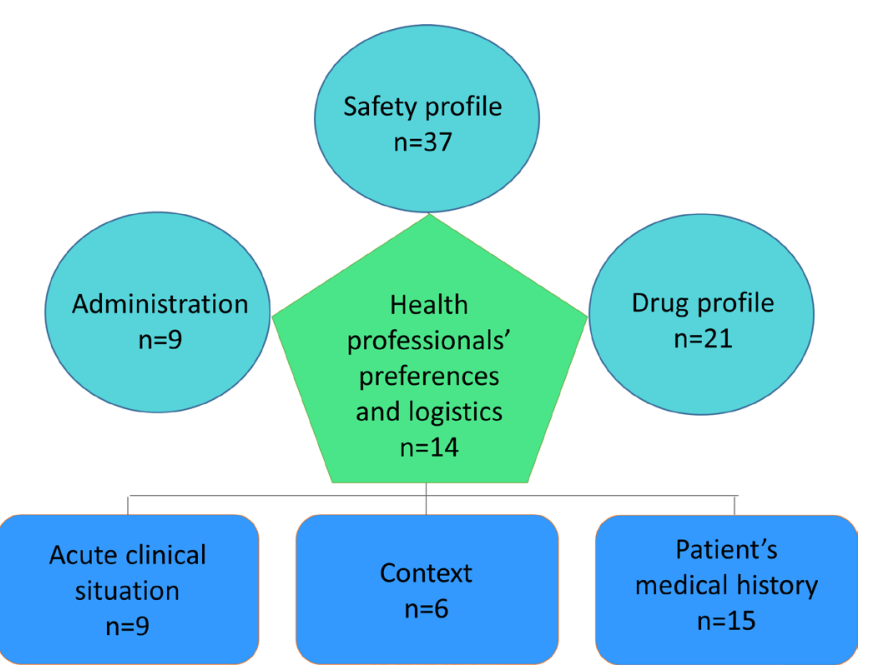

Figure 3 Conceptual model on values and preferences around choice of analgesia in emergency and prehospital settings in patients with acute trauma pain.

this table, a final conceptual model revealing values and preferences around the choice of analgesia in emergency and prehospital settings in patients with acute trauma pain was developed (figure 3). The model illustrates how three clusters related to aspects of the emergency situation (context, patient's medical history and acute clinical situation), formed a basis for the health professional's decision-making and how aspects related to various drugs (drug profile, administration and safety profile) informed the choice of analgesia.

\section{DISCUSSION}

This paper illustrates how to apply the methodology of GCM to identify, organise and prioritise the values and preferences of stakeholders, using the example of health professionals' choice of analgesia for patients with acute trauma pain in emergency and prehospital settings. Based on GCM, a conceptual model was developed presenting seven clusters or themes that included 111 unique statements on factors influencing various health professionals' choice of analgesia in acute situations.

When operationalising difficult concepts such as values and preferences of stakeholders in developing guidelines, it is important to realise that the revelation of a practical tool to assess values and preferences is made up of two important and sequential components: decisions about what to measure, the domains, and how to measure the domains. Thus, the selection or development of appropriate instruments to measure domains must take place after the domains are identified. In this study, seven individual domains were identified: drug profile, administration, context, health professionals' preferences and logistics, safety profile, patient's medical history and acute clinical situation. Based on these findings, an instrument could be developed to assess values and preferences of health professionals when choosing between analgesics, which are comparable in efficacy and safety. Such an instrument with items based on the seven domains and 111 statements could potentially yield a seven-subscale profile of scores that the guideline panel need to follow.

\section{The conceptual model}

A conceptual model represents a way to concretise a phenomenon. In this study, the phenomenon explored was what influences health professionals' choice of analgesia in a given situation. The conceptual model illustrates how each specific situation involves evaluation of the acute clinical circumstances and the patient's medical history, which form the basis for clinical decision-making. In addition, specific knowledge around drug profile and safety influences the professional's choice. By looking at the unique statements within each theme, it is possible to get a deeper understanding of what determines the choice of analgesia in an emergency situation. The model illustrates the complexity of the situations in which the health professionals need to make informed choices. The conceptual model also underlines that research evidence concerning drug effectiveness is only one of several factors involved in clinical decision-making and stresses the need to uncover and take into consideration the values and preferences of the health professionals when developing recommendations and guidance on analgesia.

\section{The value of GCM}

The conceptual model was generated through GCM aiming at identifying, organising and prioritising values and preferences among health professionals. A qualitative approach is traditionally applied to describe, understand and interpret experiences and structures within the context in which they occur. ${ }^{30}$ However, qualitative findings are limited to the specific research context and thus not generalisable to larger groups. ${ }^{30}$ One of the benefits of GCM is that perspectives and ideas can be extracted on a specific topic from a large number of stakeholders with various characteristics so that 'group wisdom' is revealed, enhancing generalisation of the results. ${ }^{14}{ }^{15}$ In general, high validity, reliability and completion percentages have been found in GCM studies. ${ }^{24}$

GCM represents a mixed methods approach with true integration of qualitative and quantitative data, ${ }^{15}$ to an extent that it has been argued that the distinction between qualitative and qualitative paradigms becomes unclear. ${ }^{14}$ The GCM process values the voice and involvement of the participants, who are involved in generating data, and in data analyses and validation of the results. Finally, a unique feature with this method is that the emerging concepts and their relationship are visualised in GCM maps. ${ }^{15}$ This feature facilitates communicating and disseminating the results to the stakeholders. The fact that a conceptual model was developed with seven inter-related themes and a high number of statements representing values and preferences of high importance among health professionals suggests that GCM may be a suitable method to assess the values and preferences among stakeholders in other settings. 
The GRADE system classifies guideline recommendations as either strong or conditional. The strength of a recommendation reflects the extent to which we can be confident that the desirable effects of an intervention outweigh the undesirable effects. ${ }^{2}$ Strong recommendations imply that most patients in their situation would want the recommended management strategy (and only a small proportion would not). Clinicians and policymakers can conclude that most patients should receive the recommended course of action and the recommendation may be adopted as a policy. The implication of a conditional recommendation is that physicians should recognise that different choices will be appropriate for different patients. Physicians must guide each patient to arrive at a management decision consistent with her or his values and preferences. As a consequence, a conditional recommendation from a guideline panel mandate shared decision-making. ${ }^{31}$ With the present study, we present a methodology that can help generate a list of items, ranked and clustered into subdomains that could help a guideline panel explicitly rank the values and preferences of relevant stakeholders. The more values and preferences vary, and uncertainty in the scores collected is evident (eg, statistical dispersion), this can be a reason to make a conditional (weak) rather than a strong recommendation. Making trade-offs between desirable and undesirable consequences of alternative management strategies is the fundamental process of making recommendations, a process that requires making value and preference judgements. ${ }^{32}$ When the benefits and harms appear very comparable, but the route of pharmacological drug administration varies considerably, value and preference judgements are particularly relevant and should accompany individual recommendations. Ideally, the values and preferences applied to a strong recommendation would represent the average values and preferences of the patient population. If we were to create a 'value and preference outcome', and knowledge of the extent to which patient values and preferences vary would be visible, it can be assumed that the greater the variability in values and preferences (eg, score dispersion in the form of IQRs), the more likely it is that a conditional recommendation is warranted.

\section{Strengths and limitations}

This study was possibly limited by a smaller sample size than anticipated. However, all five Nordic countries were represented in the brainstorming phase as well as the sorting and rating phase. In addition, participants from different disciplines within acute care took part in all phases throughout the GCM process, which secured a wide range of perspectives in the data collection. The online participation that was applied yielded a large number of statements during the brainstorming phase $(n=151)$, and the fact that 40 of the statements were redundant indicated that the number of statements was sufficient to reach data saturation. The number of participants involved in the sorting and rating stage could also, as according to Jackson and Trochim, be assumed to be sufficient, as at least 10-12 participants in general are needed to sort and rate to perform a valid statistical analysis. ${ }^{33}$ Moreover, a stress value below the commonly accepted threshold indicated that the sorting of statements was of sufficient reliability despite the relatively low number of participants. ${ }^{33}$

The validation meeting in which stakeholders came together in person is a strength in general, as this forum allowed the stakeholders to provide additional inputs and discussions to supplement and validate the results of the statistical analysis. In the process of GCM, statistical analysis is to be considered a starting point that should be applied in concert with human analyses. ${ }^{33}$ Although Sweden and Iceland were not represented at the validation meeting, participants from Denmark, Norway and Finland from different professions participated, bringing their individual perspectives to the validation of the results.

\section{Perspectives}

The conceptual model developed in this study can be used to illustrate the complexity of clinical decision-making when choosing analgesia in an emergency situation. As mentioned above, this complexity should be taken into consideration when developing clinical recommendations and guidelines. Another possibility is to develop a screening tool, based on a selected subset of the identified statements, to assess if requirements of values and preferences are met with any analgesic product used in prehospital acute situations.

Although this study specifically concerns the values and preferences of health professionals dealing with acute patients in the field of pain treatment, the GCM method could be applied in any setting to assess the specific values and preferences of stakeholders within a certain field.

\section{CONCLUSION}

Based on GCM important values and preferences were identified around the choice of analgesia in patients with acute trauma pain. The approach proved to be a valid method to assess the values and preference criterion in the GRADE evidence to decision framework, with the example of analgesia for acute trauma pain. The conceptual model can support health professionals in their decision-making when choosing the best available treatment for acute pain in emergency care.

\section{Author affiliations}

${ }^{1}$ Department of Occupational Therapy, University College of Northern Denmark (UCN), Aalborg, Denmark

${ }^{2}$ The Parker Institute, Bispebjerg and Frederiksberg Hospital, University of Copenhagen, Copenhagen, Denmark

${ }^{3}$ Department of Rheumatology, Odense University Hospital, Odense, Denmark ${ }^{4}$ The Research Initiative for Activity Studies and Occupational Therapy, General Practice, Department of Public Health, University of Southern Denmark, Odense, Denmark

Acknowledgements The authors thank the health professionals who participated in the study. 
Contributors RC, LK and MUR generated the idea for this study, and KTN, MUR and EEW designed the study, acquired the data and participated in the interpretation of the data together with AFO. KTN and EEW performed the concept analysis. KTN, MUR, RC and EEW drafted the manuscript, while LK and AFO critically revised it for important intellectual content. All authors approved the final manuscript.

Funding This study was supported by an unrestricted grant from Mundipharma and by the Oak Foundation (OCAY-13-309). Research and Development representatives from Mundipharma have been invited to comment on the protocol (before contacting any external stakeholders and before collection of any data), on aspects such as clarity and potential outcomes, and so on. However, the process of collecting data and writing of the manuscript was done completely without interaction from Mundipharma.

Competing interests None declared.

Patient consent for publication Not required.

Provenance and peer review Not commissioned; externally peer reviewed.

Data availability statement All data relevant to the study are included in the article or uploaded as supplementary information. All statements on which the analysis is based are displayed in a supplementary file.

Open access This is an open access article distributed in accordance with the Creative Commons Attribution Non Commercial (CC BY-NC 4.0) license, which permits others to distribute, remix, adapt, build upon this work non-commercially, and license their derivative works on different terms, provided the original work is properly cited, appropriate credit is given, any changes made indicated, and the use is non-commercial. See: http://creativecommons.org/licenses/by-nc/4.0/.

\section{ORCID iDs}

Kristina Tomra Nielsen http://orcid.org/0000-0002-4944-9453

Marianne Uggen Rasmussen http://orcid.org/0000-0001-9257-3073

\section{REFERENCES}

1 Coffey F, Dissmann P, Mirza K, et al. Methoxyflurane analgesia in adult patients in the emergency department: a subgroup analysis of a randomized, double-blind, placebo-controlled study (stop!). Adv Ther 2016;33:2012-31.

2 Guyatt GH, Oxman AD, Kunz R, et al. Going from evidence to recommendations. BMJ 2008;336:1049-51.

3 Alonso-Coello P, Oxman AD, Moberg J, et al. Grade evidence to decision (ETD) frameworks: a systematic and transparent approach to making well informed healthcare choices. 2: clinical practice guidelines. BMJ 2016;353:i2089.

4 Zhang Y, Coello PA, Brożek J, et al. Using patient values and preferences to inform the importance of health outcomes in practice Guideline development following the grade approach. Health Qual Life Outcomes 2017;15:52-621.

5 Chong CAKY, Chen I-je, Naglie G, et al. How well do guidelines incorporate evidence on patient preferences? J Gen Intern Med 2009;24:977-82.

6 Krahn M, Naglie G. The next step in Guideline development: incorporating patient preferences. JAMA 2008;300:436-8.

7 Montori VM, Brito JP, Murad MH. The optimal practice of evidencebased medicine: incorporating patient preferences in practice guidelines. JAMA 2013;310:2503-4.

8 Ubel PA. Medical Facts versus Value Judgments--Toward Preference-Sensitive Guidelines. N Engl J Med 2015;372:2475-7.

9 MacLean S, Mulla S, Akl EA, et al. Patient values and preferences in decision making for antithrombotic therapy: a systematic review: antithrombotic therapy and prevention of thrombosis, 9th ED: American College of chest physicians evidence-based clinical practice guidelines. Chest 2012;141:e1S-23.
10 Tarabay R, El Rassi R, Dakik A, et al. Knowledge, attitudes, beliefs, values, preferences, and feasibility in relation to the use of injection safety devices in healthcare settings: a systematic review. Health Qual Life Outcomes 2016;14:102.

11 Poremski D, Sagayadevan Vathsala D/O, Wang P, et al. The impact of Stakeholder preferences on service user adherence to treatments for schizophrenia and metabolic comorbidities. PLoS One 2016;11:e0166171

12 Bastemeijer CM, Voogt L, van Ewijk JP, et al. What do patient values and preferences mean? A taxonomy based on a systematic review of qualitative papers. Patient Educ Couns 2017;100:871-81.

13 Guyatt GH, Norris SL, Schulman S, et al. Methodology for the development of antithrombotic therapy and prevention of thrombosis guidelines: antithrombotic therapy and prevention of thrombosis, 9th ED: American College of chest physicians evidence-based clinical practice guidelines. Chest 2012;141:53S-70.

14 Kane M, Trochim WM. Concept mapping for planning and evaluation. 1st ed. London: Sage Publications, Inc, 2007.

15 Kane M, Rosas S. Conversations about group concept mapping, applications, examples and enhancements. 1st ed. Thousand Oaks, California: Sage Publications, Inc, 2018.

16 Trochim W, Kane M. Concept mapping: an introduction to structured conceptualization in health care. Int J Qual Health Care 2005; 17:187-91.

17 Trochim WM, Cook JA, Setze RJ. Using concept mapping to develop a conceptual framework of staff's views of a supported employment program for individuals with severe mental illness. J Consult Clin Psychol 1994;62:766-75.

18 Nielsen KT, Klokker L, Guidetti S, et al. Identifying, organizing and prioritizing ideas on how to enhance ADL ability. Scand J Occup Ther 2019;26:1-12.

19 Hackett KL, Lambson RL, Strassheim V, et al. A concept mapping study evaluating the UK's first NHS generic fatigue clinic. Health Expect 2016;19:1138-49.

20 Hargett CW, Doty JP, Hauck JN, et al. Developing a model for effective leadership in healthcare: a concept mapping approach. $J$ Healthc Leadersh 2017;9:69-78.

21 Trochim WMK, Milstein B, Wood BJ, et al. Setting objectives for community and systems change: an application of concept mapping for planning a statewide health improvement initiative. Health Promot Pract 2004;5:8-19.

22 Ogden K, Barr J, Greenfield D. Determining requirements for patientcentred care: a participatory concept mapping study. BMC Health Serv Res 2017;17:780-2741.

23 Nielsen KT, la Cour K, Christensen JR, et al. Lessons learned about occupation-focused and occupation-based interventions: a synthesis using group concept mapping methodology. Scand J Occup Ther 2019;20:1-12.

24 Rosas SR, Kane M. Quality and rigor of the concept mapping methodology: a pooled study analysis. Eval Program Plann 2012;35:236-45

25 Marshall MN. Sampling for qualitative research. Fam Pract 1996;13:522-6.

26 Survey Xact online survey system [computer program]. Version 2013. 2013.

27 CSGlobal MAX online software [computer program]. 2005.

28 Microsoft Office Excel [computer program]. 2010.

29 De Vidensskabsetiske Komiteer. Komitéloven § 1, STK. 4. 2012. Ref type: statute.

30 DePoy EGLN. Introduction to research, understanding and applying multiple strategies. 4th ed. St. Luis, Missouri: Elsevier/Mosby, 2011.

31 Guyatt G. Grade weak or conditional recommendations mandate shared decision-making. J Clin Epidemiol 2018;18:10.

32 Sackett DL, Rosenberg WM, Gray JA, et al. Evidence based medicine: what it is and what it isn't. BMJ 1996;312:71-2.

33 Jackson KM, Trochim WMKConcept mapping as an alternative approach for the analysis of open-ended survey responses. Organ Res Methods 2002;5:307-36. 